\title{
Tinea capitis in 2 immunocompetent elderly patients
}

Keywords: tinea capitis, trychophyton tonsurans, elderly, folliculitis, corticosteroids

\section{Introduction}

Tinea capitis is classically considered as a childhood disease, however in recent years has been an increase in the involvement of adults. The prevalence of tinea capitis in adults is around $10 \%$ in southern of Spain. ${ }^{1,2}$ The population have been epidemiological that had conditioned the emergence of etiological agents in fluctuating form. ${ }^{3}$ We review the frequency of Trichophyton tonsurans in our hospital center and present 2 immunocompetent elderly patients with tinea capitis in whom this agent is isolated.

\section{Objective}

Determine the prevalence of tinea capitis due to Trichophyton tonsurans in the elderly population of the University Hospital of La Princesa, Madrid.

\section{Materials and methods}

A retrospective study was performed since 2009 to 2016. It were reviewed all the positive fungal samples of skin lesions and annexes sent to microbiology.

\section{Results}

In total, 424positive samples for fungi with skin lesions and adnexa were collected. From these only 6 presented a culture of Trichophyton tonsurans, which corresponds to $1.4 \%$ of the total positive cultures. In 3 of the 6 cases had tinea capitis and 2 from the 3 patients were older than 65 years.

The first case is an 87year-old woman, without immunosuppressive factors and without risk environment, who started 18 months before with pruritic lesions on the scalp, alopecia, erythema, thick adherent scales, pustules and follicular black dots (Figure 1A) (Figure 1B). Fungal culture was positive for Trichophyton tonsurans. Serologies (VHB, VHC, and VIH) were negative and a complete blood count test and biochemistry analysis was normal. Histopathology showed a follicular involvement by a dermatophyte with an endothrix pattern (Figure 2A). Terbinafine $250 \mathrm{mg}$ /day was prescribed for 16 weeks. A persistence of residual slight erythema was observed. Control cultures and $\mathrm{KOH}$ were negatives.

The second case is a 90year-old woman, without immunosuppressive factors and without a risk environment, which began 6months before with progressive pruritus on the scalp, with diffuse erythema, follicular hyperkeratosis, pustules and diffuse black dots. Numerous annular and circinate erythematous and squamous plaques were observed in the dorsal neck and scapular region (Figures 3A-3C). Fungal culture was positive for Trichophyton tonsurans. Serologies (VHB, VHC, and VIH) were negative and a complete blood count test and biochemistry analysis was normal. Histopathology showed a follicular involvement by a dermatophyte with an endothrix pattern (Figure 2B). Itraconazole $200 \mathrm{mg}$ /day for 8 weeks and eberconazole $1 \%$ topical was prescribed with progressive disappearance of the lesions.

\author{
Volume 2 Issue 2 - 2018
}

\author{
Martinez-Mera C,' Capusan TM,' Moyano- \\ Herrero M,' Gordillo-Vélez C,' 'Granja \\ Torrecillas S, ${ }^{3}$ Sánchez Pérez J' \\ 'Department of Dermatology, Hospital Universitario de La \\ Princesa, Spain \\ ${ }^{2}$ Department of Pathology, Hospital Universitario de La \\ Princesa, Spain \\ ${ }^{3}$ Department of Microbiology, Hospital Universitario de La \\ Princesa, Spain
}

Correspondence: Constanza Martinez Mera, Department of Dermatology, Diego de León 62, Postal code 28006, Madrid, Spain, Tel +34608771 695, Email c.martinezmera@gmail.com

Received: March II, 2018| Published: April 10, 2018

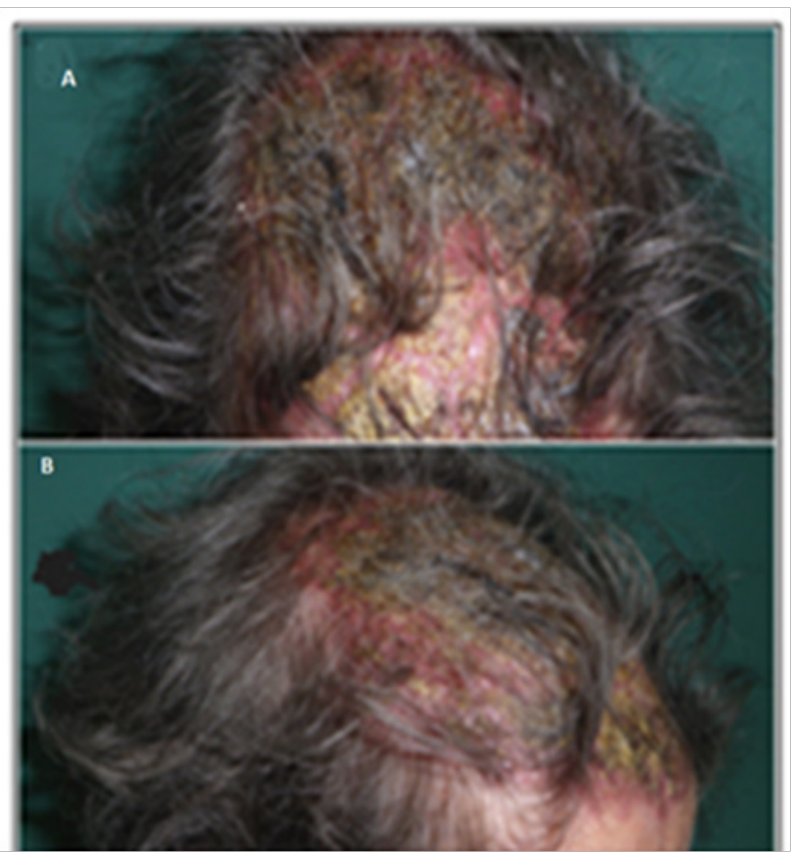

Figure I(A,B) Tinea capitis with alopecia in bilateral frontal and parietal region. Erythema, thick crust adhered, pustules and follicular black dots.

\section{Discussion}

In recent years Trichophyton tonsurans is becoming increasingly isolated, linked to immigration from Africa, among other factors. ${ }^{2,3}$ Related to our local epidemiology, it was observed that in the period 2009-2016 there was a low prevalence of Trichophyton tonsurans, which could be explained by the limited foreign population that is treated in our hospital. Tinea capitis in adults predominate in postmenopausal women, prone by the use of topical corticosteroids. ${ }^{4}$ 
Desquamative lesions, alopecia and black dots have traditionally been related to infection by Trichophyton tonsurans. ${ }^{5,6}$ Exceptionally cases have been reported where the predominance of inflammatory lesions are seen as in the first case. ${ }^{7}$

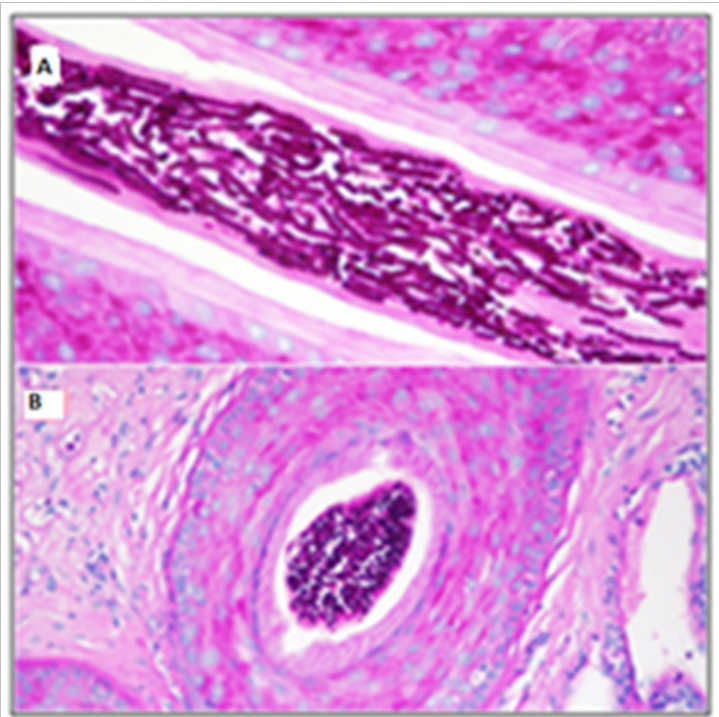

Figure 2 (A) Tinea capitis with endothrix pattern first case HE (Hematoxilin and eosin) $\times 40$.

(B) Tinea capitis with endothrix pattern second case HEx20.

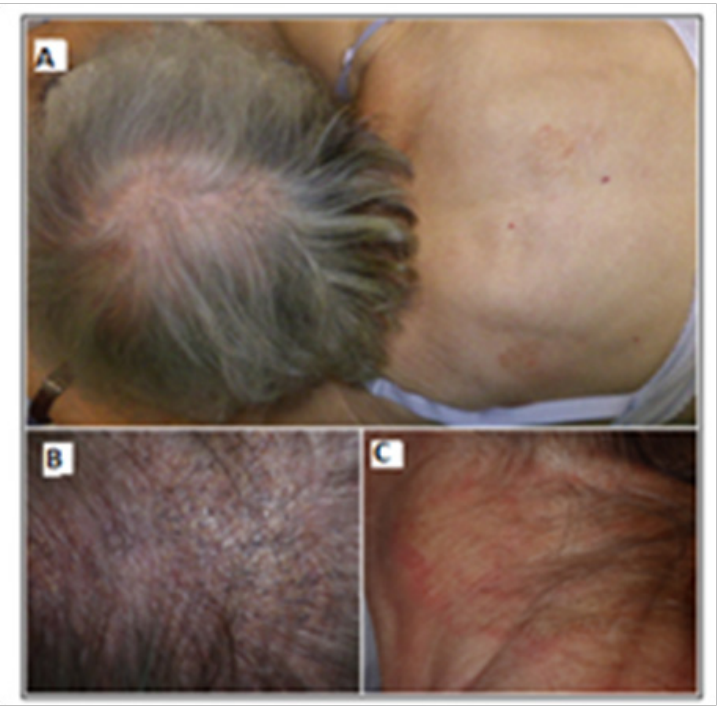

Figure 3 (A) Tinea capitis and corporis. (B) Diffuse erythema, follicular hyperkeratosis, pustules, and diffuse black dots on the scalp. (C) Erythematous and squamous annular and circinates plaques in cervical region.
It is important to exclude fungal infections in cases that simulate seborrheic dermatitis, folliculitis or impetigo with torpid evolution, because produce a delate in the diagnosis, as in our patient who being delayed a year and a half after the start of the clinic. Mostly is important to perform cultures prior to treatments, to make the right diagnosis, identify the correct etiological agent and know adequately the local epidemiology of tinea capitis of each center.

\section{Acknowledgements}

None.

\section{Conflicts of interest}

This manuscript has not been published elsewhere and is not under consideration by another journal. All authors have approved the manuscript and agree with submission to the Journal of Dermatology $\&$ Cosmetology. The authors have no conflicts of interest to declare.

\section{References}

1. Lova-Navarro M, Gómez-Moyano E, Martínez Pilar L, et al. Tinea capitis in adults in southern Spain. A 17-year epidemiological study. Rev Iberoam Micol. 2016;33(2):110-113.

2. García-Martos P, García-Agudo L, Agudo-Pérez E, et al. Dermatophytoses due to anthropophilic fungi in Cadiz, Spain, between 1997 and 2008. Actas Dermosifiliogr. 2010;101(3):242-247.

3. Del Boz-González J. Tendencias de la tinea capitis en España. Actas Dermosifiliogr. 2012;103(4):288-293.

4. Khosravi A, Shokri H, Vehedi G. Factors in Etiology and predisposition of adult tinea capitis and review of published literature. Mycopathologia. 2016;181(5-6):371-378.

5. Rebollo N, López-Barcenas AP, Arenas R. Tinea Capitis. Actas Dermosifiliogr. 2008;99(2):91-100.

6. Morell L, Fuente MJ, Boada A, et al. Tinea capitis en mujeres de edad avanzada: descripción de 4 casos. Actas Dermosifiliogr. 2012;103(2):144-148.

7. Buckley D, Fuller L, Higgins E, et al. Tinea Capitis in adults. BMJ. 2000;320(7246):1389-1390. 